\title{
Oscillation-Based Test in a CCII-based Bandpass Filter
}

\author{
Pablo Petrashin, Luis Toledo, Walter Lancioni \\ Laboratorio de Microelectrónica \\ Universidad Católica de Córdoba \\ Córdoba, Argentina
}

\author{
Piotr Osuch, Tinus Stander \\ Carl and Emily Fuchs Inst. for Microelectronics \\ Dept. EEC Engineering - University of Pretoria \\ Pretoria, South Africa
}

\begin{abstract}
Oscillation based testing (OBT) has proven to be a simple yet effective VLSI test for numerous circuit types. In this work, OBT is applied to test Second-generation Current Conveyor (CCII) based filters for the first time. Adopting a CCII-based band pass filter as a case study, it is shown that OBT can be implemented with a minimally intrusive switched feedback loop to establish the oscillator. Exhaustive fault simulation indicates $98.11 \%$ detection of possible short circuit and $100 \%$ detection of possible open circuit faults in the circuit under test, in both $0.35 \mu \mathrm{m}$ and $1.2 \mu \mathrm{m}$ CMOS technology nodes.
\end{abstract}

Keywords - Oscillation based test; continuous time filters; analog testing; fault simulation; testing

\section{INTRODUCTION}

The Oscillation-Based Test is a Design-for-Test technique that can be used either in offline testing or as the core of the so-called OBIST (Oscillation-Based Built-In-Self-Test). The basic idea is to switch the Circuit Under Test (CUT) into an oscillator configuration during the test phase, as has been extensively discussed for several circuits (operational amplifiers, filters, data converters) [1-3]. During the test, a feedback loop is created to the CUT to produce self-sustained oscillations. This feedback can be globally applied to the overall CUT or to a part of it. Faulty behavior is then indicated by deviations in the frequency and / or the amplitude of the oscillations when compared to the values of these test attributes under fault-free conditions [2]. OBT avoids the problem of test vector generation, requires relatively simple measurements and generally does not demand circuit modifications during the testing phase. These characteristics make OBT an appealing strategy.

The implementation of OBT depends heavily on the characteristics of the system under test. The oscillator has to be designed and implemented based on several considerations, such as the characteristics of the system, the possibility of partitioning the CUT, the observable outputs, the analog or digital nature of the signal, etc. Consequently, the application of this strategy to each new class of circuits becomes a challenging task.

The test of low-order continuous-time filters by OBT can be found in [3-5]. In these papers, simple and relatively common topologies are adopted, and analytical expressions for the oscillation conditions are obtained. Huertas et al. [6 - 8]

This work was supported by the Argentina - South Africa Research Cooperation Programme, as administered by the Ministry of Science, Technology and Productive Innovation in Argentina and the National Research Foundation in South Africa. and Kac and Novak [9] have studied the application of OBT to high-order switched-capacitors filters designed as cascade of first- and second-order sections. These authors propose to subdivide the filters into lower-order sections and to test each section separately. Although this approach could help identify individual failure points, the specific point of failure is not important from a pass / fail test of the entire IC. Furthermore, the extra circuitry needed to switch the circuit from test mode to normal mode may cause unacceptable degradation in the signal. Finally, the method cannot be extended to filters that cannot be divided in low-order sections.

In this work, we explore the ability of OBT for testing CCII-based filters for the first time. Adopting an oscillator proposed by [10] as a case study, we present a scheme that makes use of a linear characteristic in the feedback loop, as seen in Fig. 1. The evaluation of the test quality is made by means of fault simulation.

\section{FILTER UNDER TEST}

Despite the extensive use of digital signal processing in modern electronics, analogue front-ends and active filters are still used extensively in mixed-signal ICs. The second generation current conveyor (CCII) is an adaptable multifunction component that can be applied in a large number of analogue circuits (such as active continuous time filters and all-pass networks), and has been the subject of intensive study for the last decade [11 - 19]. Most CCII-based circuits do not require bulky on-chip inductors and only use transistors, resistors and capacitors, making them cost attractive solutions. Additionally, the component count of these structures is usually very low, further decreasing the area cost [11].

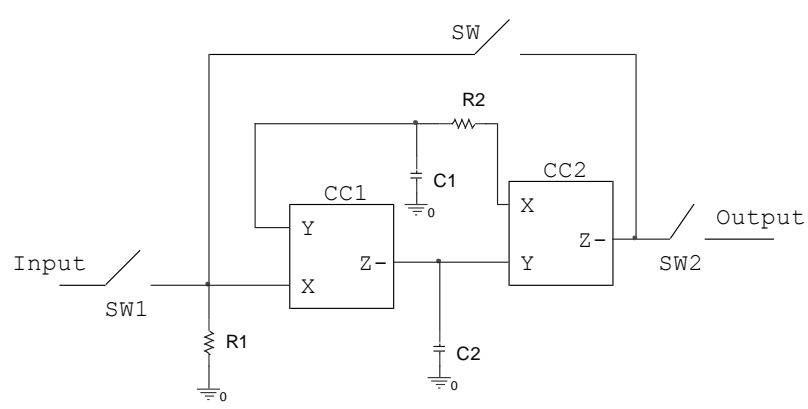

Fig. 1. Block diagram of the filter under study 


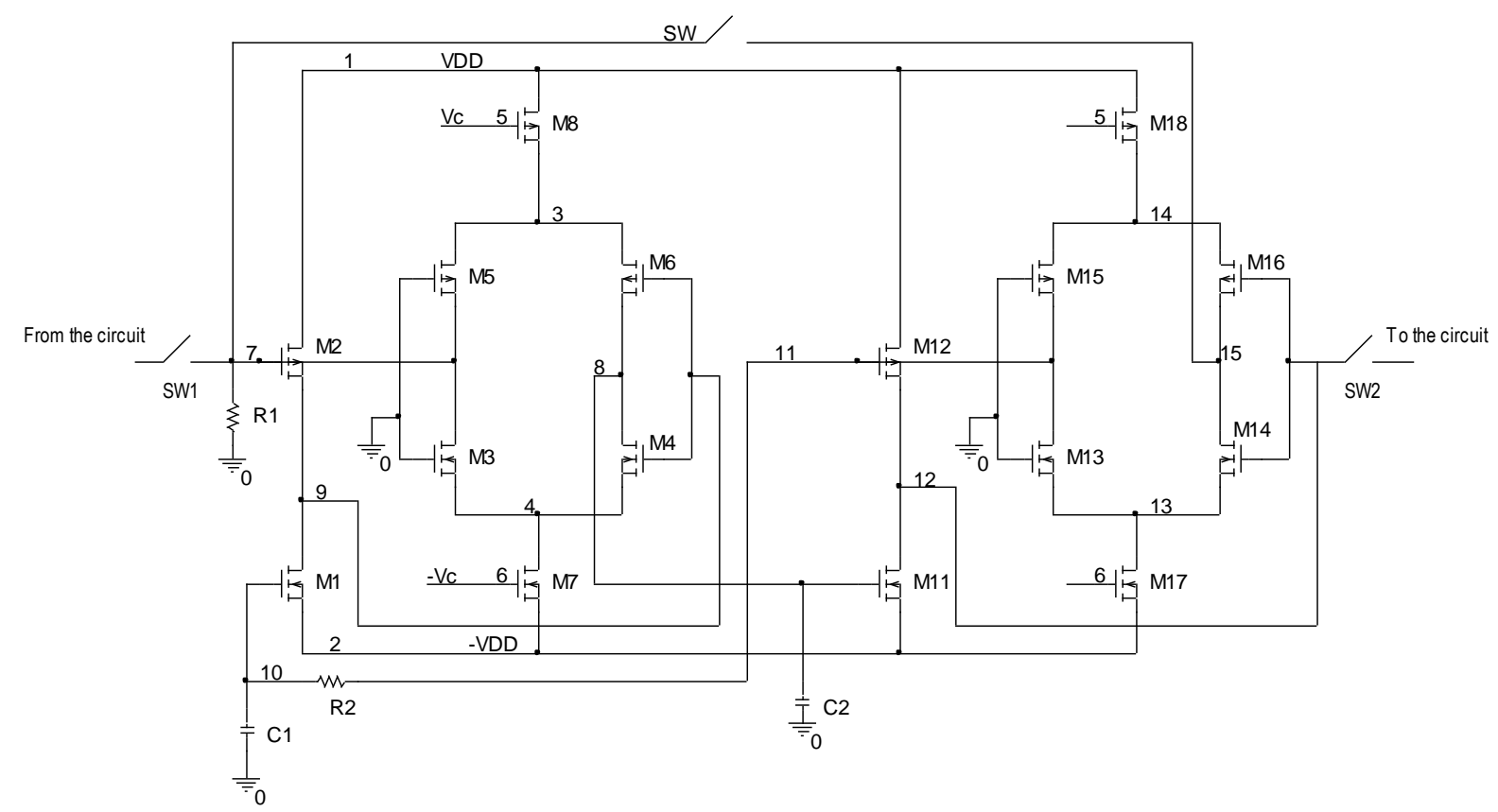

Fig. 2. Transistor level schematic of the filter under test

TABLE I. COMPONENT VALUES FOR THE CIRCUIT OF FIGURE 2.

\begin{tabular}{|l|l|l|l|}
\hline M1, M11 & $30 / 4.8 \mu \mathrm{m}$ & M8, M18 & $198 / 3.6 \mu \mathrm{m}$ \\
\hline M2, M12 & $97.2 / 4.8 \mu \mathrm{m}$ & R1 & $750 \Omega$ \\
\hline M3, M4, M13, M14 & $60 / 1.2 \mu \mathrm{m}$ & R2 & $1500 \Omega$ \\
\hline M5, M6, M15, M16 & $120 / 1.2 \mu \mathrm{m}$ & $\mathrm{C} 1$ & $500 \mathrm{pF}$ \\
\hline M7, M17 & $94.8 / 3.6 \mu \mathrm{m}$ & $\mathrm{C} 2$ & $500 \mathrm{pF}$ \\
\hline
\end{tabular}

In order to explore the effectiveness of OBT for these structures, a minimum passive component pass-band filter is adopted as a case study. The basic block diagram of this filter is shown in Fig. 1; when switch SW is closed, the filter is fed back and placed into an oscillation condition. Switches SW1 and SW2 are used to isolate the CUT from the rest of the circuitry on chip.

The CCII used in this filter is presented in [15], with [16] providing an in-depth OBT study of the CCII in isolation. The transistor-level schematic of the filter is depicted in Fig. 2, with the component values given in Table 1. A simulation of the design is performed in two different technology nodes: AMS C35 $0.35 \mu \mathrm{m}$ CMOS process, and the technology proposed in [15], AMI $1.2 \mu \mathrm{m}$. The frequency response of the filter under test (SW open) is shown in Fig. 3, for both the C35 and AMI 1.2um technologies.

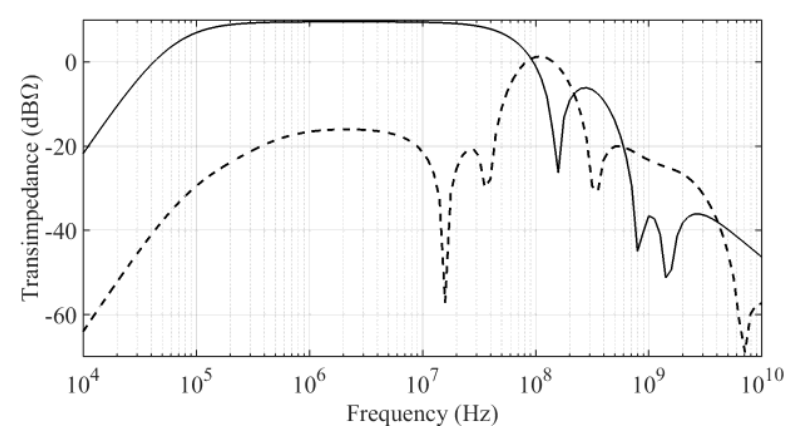

Fig. 3. Magnitude response for C35 (dotted) and AMI 1.2 um (solid)

\section{OBT IMPLEMENTATION}

Converting the filter into an oscillator requires a mechanism to force the oscillation condition. In circuits with a clear pass-band with positive gain and sufficient phase shift [10], only one switch, SW, is required to accomplish this condition (as shown in Figs. 1 and 2). When this switch is closed, the circuit begins to oscillate.

With a slight change in biasing conditions, the circuit has been proven to oscillate in both technologies, so OBT has been applied twice, one for each technology. The simulated output waveforms for the non-faulty oscillator in both technology processes are presented in Fig. 4. An oscillation frequency $f_{0}$ of $300 \mathrm{kHz}$ for both technology nodes and amplitudes $A$ of $250 \mathrm{mV}_{\mathrm{pk}-\mathrm{pk}}$ for the $0.35 \mu \mathrm{m}$ process and 44 $\mathrm{mV}_{\mathrm{pk}-\mathrm{pk}}$ for the $1.2 \mu \mathrm{m}$ CMOS node can be observed. 


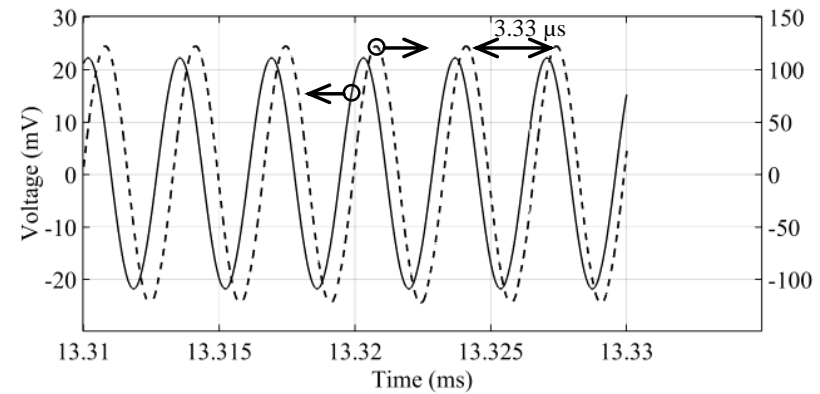

Fig. 4. Oscillator output under non faulty condition for C35 (dotted) and AMI 1.2 um (solid)

As shown in Fig. 1, only the input and output nodes of the circuit are manipulated in order to place the CUT into the oscillation mode. The circuit under test is, therefore, not modified to reach the oscillation condition, allowing an extremely low level of intrusion in the circuit and minimizing the signal degradation in the normal operating mode.

\section{FAULT MODELING}

Traditionally, the efficiency of OBT has been evaluated at structural level by using single-catastrophic and singledeviation fault-models. In this way, it is possible to use the well-known metric of fault-coverage for qualifying the test [17-19]. In this work, we focus our attention on catastrophic faults at both device and circuit level. An exhaustive fault list from the schematic is generated, considering only catastrophic faults (as is expected in production errors).

This fault list includes all possible open and short faults of transistors, with the only exception being the gate contact open fault, since this fault causes no changes in the circuit behavior. We consider short faults at the circuit level in order to take into account all probable faults. This procedure allows further comparisons with results reported by other authors.

The CUT has 15 nodes. The main nodes and schematically redundant ones are listed in Table 2 and labeled in Fig. 2. These reference and schematically redundant nodes are connected to the same potential, making it impossible to detect behavioral differences between them. Due to this fact, for short circuit simulations, only the reference nodes of Table 2 are considered. Of course, schematically redundant nodes are not necessarily also physically redundant, in the sense that different behavior of the CUT could be obtained if an open fault is located in different schematically redundant nodes. Therefore, for open circuit faults, all nodes in this table are considered separately (excluding the gate open contact fault, as is pointed out above). Using these rules, there are 36 possible open circuits, and taking into account all redundant nodes, there are 851 possible short circuits in the CUT.

Fault simulations are carried out in SPICE considering only single fault injection. Open circuit faults are modeled by a $10 \mathrm{M} \Omega$ resistor while short circuits are modeled by a $10 \Omega$ resistor. It is assumed here that a fault is detected when, the circuit ceases to oscillate or either the oscillation frequency $f_{0}$ or amplitude $A$ are out of a tolerance band, defined as $\pm 5 \%$ of the free-fault oscillator frequency. This latter means that, if a given CUT presents a frequency and/or amplitude deviation of less than 5\% from the nominal values in Fig. 3, the circuit is passed as fault-free. This margin has previously shown to account adequately for process variation [16-19] without passing faulty circuits.

TABLE II. REFERENCE NODE AND REDUNDANT NODES

\begin{tabular}{|c|c|}
\hline $\begin{array}{l}\text { Reference } \\
\text { node }\end{array}$ & Schematically redundant nodes \\
\hline 1 & M2_S, M8_S, M12_S, M18_S \\
\hline 2 & M1_S, M7_S, M11_S, M17_S \\
\hline 3 & M8_D, M5_S, M6_S \\
\hline 4 & M3_S, M4_S, M7_D \\
\hline 5 & M8_G, M18_G \\
\hline 6 & M7_G, M17_G \\
\hline 7 & M2_G, R1_1, Rfeed_1 \\
\hline 8 & M6_D, M4_D, M11_G, C2_1 \\
\hline 9 & M2_D, M1_D, M6_G, M4_G \\
\hline 10 & M1_G, R2_1, C1_1 \\
\hline 11 & R2_2, M12_G \\
\hline 12 & M12_D, M11_D, M16_G, M14_G \\
\hline 13 & M13_S, M14_S, M17_D \\
\hline 14 & M15_S, M16_S, M18_D \\
\hline 15 & M14_D, M16_D, Rfeed_2 \\
\hline
\end{tabular}

Tables 3 and 4 present the simulation results for short circuit and open circuit faults, respectively, both for $0.35 \mu \mathrm{m}$ CMOS. Faults that result in a loss of oscillation altogether are not presented in the tables. Sixteen short circuit faults were not detected by the test procedure, representing $1.88 \%$ of the whole set. It should be said, however, that these non-detected faults are short circuits between Vdd and Vss, which form a $10 \Omega$ resistance parallel to the otherwise functioning CUT. While these faults will not alter the behavior of the CUT, it could be detected by the resulting increase in supply current through an IDDQ test.

The rate was found to be the same for $1.2 \mu \mathrm{m}$ CMOS. This means that the test obtained $98.11 \%$ efficiency for detecting catastrophic shorts. In both technologies used, all 32 open circuit faults are detected, making the test $100 \%$ efficient.

For $1.2 \mu \mathrm{m}$ CMOS, all faults, with the sole exception of Vdd-Vss short circuits, resulted in a loss of oscillation. For $0.35 \mu$ CMOS, in almost all cases of detected faults (M16_D open fault being the exception), both the frequency and the amplitude of the measured signal are outside the fault-free limits. This would indicate (as it already was noticed in previous work [17-19]) that OBT can be effectively applied to this circuit using only one of the two output values.

As it was previously pointed out, it has been observed in the simulation experiments, for both technologies, that there are some faults (i.e. short circuits between Vdd and Vss) that 
do not affect the oscillation frequency or the amplitude but they have an easily noticeable effect in the IDD current. This would suggest that the implementation of an IDDQ testing would increase the fault coverage. However, this would also increase the circuit complexity and test subsequent cost. If, however, it is implemented, IDDQ testing would detect all of the short circuit faults currently undetected by amplitude or frequency measurement. This result would increase the fault coverage to $100 \%$. For the open circuit faults, however, the technique would not improve the fault coverage, if needed.

TABLE III. SHORT CIRCUIT SIMULATION RESULTS

\begin{tabular}{|l|l|l|}
\hline NA_NB & $\Delta \mathrm{f} / \mathrm{f}_{\text {ref }}(\%)$ & $\Delta \mathrm{A} / \mathrm{A}_{\text {ref }}(\%)$ \\
\hline $10--14$ & $-20,64 \%$ & $12,67 \%$ \\
\hline $11--12$ & $-38,17 \%$ & $130,16 \%$ \\
\hline $11--14$ & $-12,28 \%$ & $-17,39 \%$ \\
\hline $10--11$ & $733,33 \%$ & $-85,71 \%$ \\
\hline
\end{tabular}

TABLE IV. OPEN CIRCUIT SIMULATION RESULTS.

\begin{tabular}{|l|l|l|}
\hline Node & $\Delta \mathrm{f} / \mathrm{f}_{\text {ref }}(\%)$ & $\Delta \mathrm{A} / \mathrm{A}_{\text {ref }}(\%)$ \\
\hline M16_D & $-4,77 \%$ & $-33,48 \%$ \\
\hline M16_S & $-14,53 \%$ & $-4,38 \%$ \\
\hline
\end{tabular}

\section{CONCLUSIONS}

An OBT scheme has been applied to a CCII-based band pass filter for the first time, in two different technology nodes. For evaluating OBT, we adopted catastrophic fault models at both device and circuit level. The fault simulation results show $98.11 \%$ fault coverage for short circuits, which could be improved to $100 \%$ by including additional IDDQ testing. Complete coverage is obtained for open circuit faults. These conclusions were obtained for two different technology processes, showing extremely good data consistency and proving OBT is a very effective method for testing CCII band pass filters.

It has also been shown that in almost all cases (apart from one open circuit fault) only one parameter (amplitude or frequency) is necessary to achieve the above fault coverage. This would suggest that it is possible to reduce the test circuit complexity and consequently the testing cost.

\section{ACKNOWLEDGEMENT}

Special thanks are given to Eng. Carlos Viale and Mr. Guido Righetti, both from Universidad Católica de Córdoba, for their special contribution and help with simulation procedure.

\section{REFERENCES}

[1] K. Arabi and B. Kaminska, "Oscillation-Test Strategy for analog and mixed-signal integrated circuits," Proc. 14th VLSI Test Symposium, 1996, pp. 476-482.

[2] K. Arabi and B. Kaminska, "Testing analog and mixed-signal integrated circuits using oscillation-test method," IEEE Trans. on Computer-Aided Design of Integrated Circuits and Systems, vo1.16, pp. 745-753, July 1997.

[3] K. Arabi and B. Kaminska, "Oscillation-test methodology for low-cost testing of active analog filters" IEEE Trans. on Inst. and Meas., vol. 48, pp. 798-806, August 1999.

[4] M. Santo, F. Novak and S. Macek, "Design of oscillation based test structures for active RC filters," IEE Proc. Circuits Devices Syst., vol. 147 , pp. 297-302, 2000.

[5] M. Wong, "On the issues of oscillation test methodology," IEEE Trans. on Inst. and Meas., vol. 49, pp. 240-245, 2000.

[6] G. Huertas, D. Vázquez, E. Peralías, A. Rueda and J. Huertas, "Practical oscillation-based test of integrated filters," IEEE Design \& Test of Computers, vol. 19, pp. 64-72, November- December 2002.

[7] G. Huertas, D. Vázquez, E. Peralías, A. Rueda and J. Huertas, "Testing mixed-signal cores: a practical oscillation-based test in an analog macrocell," IEEE Design \& Test of Computers, vol. 19, pp. 73-82, November- December 2002.

[8] G. Huertas, D. Vázquez, A. Rueda and J. Huertas, "Simple and secure start-up circuitry for oscillation-based test application," Analog Integrated Circuits and Signal Processing, vol. 32, pp. 187-190, 2002.

[9] U. Kac and F. Novak, "All-pass SC biquad reconfiguration scheme for oscillation-based analog BIST," Proc. 9th IEEE European Test Symposium, pp. 133-138, May 2004.

[10] Ahmed Soliman, "Generation of oscillators from current mode bandpass filters using single output ICCII", J. of Active and Passive Electronic Devices, Vol. 6, pp. 251-264, 2011.

[11] Raj Senani, D. R. Bhaskar, A. K. Singh, Current Conveyors. Variants, Applications and Hardware Implementations, 2015 Springer International Publishing.

[12] Pandey N, Kumar P, Choudhary J (2013) Current controlled differential difference current conveyor transconductance amplifier and its application as wave active filter. ISRN Electron Article ID 968749:11

[13] A. M. Ismail, A. M. Soliman, Wideband CMOS Current Conveyor, Electronics Letters, pp. 2368- 2369, 1998.

[14] S. B. Salem, M. Fakhfakh, D. S. Masmoudi, M. Loulou, P. Loumeu, N. Masmodi, A High Performances CMOS CCII and High Frequency Applications, Analog Integrated Circuits and Signal Processing, pp. 7178, 2006.

[15] Awad and Soliman, "Inverting second generation current conveyors: the missing building blocks, CMOS realizations and applications”, INT. J. ELECTRONICS, 1999, VOL. 86, NO. 4, 413- 432.

[16] P. Petrashin, L. Toledo, W. Lancioni, P. Osuch, T. Stander, "Oscillation Based Test applied to a wideband CCII", submitted for publication.

[17] Pablo Petrashin, G. Peretti, E. Romero "OBT implementation on a GmC Pass Band Filter with low gm”, 2010 11th ISQED, San José, CA, US, 22-24 March 2010 pages: 271 - 276

[18] Pablo Petrashin, Carlos Dualibe, "An improved OBT strategy for untuned continuous-time analog filters". LATW, Porto de Galinhas, Brazil, march 27-30 of 2011.

[19] P. Petrashin, C. Dualibe, “An improved OBT strategy for catastrophic faults detection in continuous-time analog filters", Argentine School of Micro-Nanoelectronics, Technology and Applications (EAMTA) 2012, pp. 62-65. 